Instability, Chaos and Predictability in Celestial Mechanics and

Stellar Dynamics
Copyright $\odot 1992$ by Nova Science Publishers, Inc. All rights of reproduction in any form reserved. ISBN 1-56072-054-9

\title{
APPLICATION OF NON-STANDARD NUMERICAL METHODS TO SATELlite ProblemS
}

\author{
M. Teresa Pérez \\ Departamento de Matemática Aplicada a la Ingeniería, E.T.S. de Ingenieros \\ Industriales, Universidad de Valladolid, 47011 Valladolid
}

\begin{abstract}
In this paper we have tested some non-standard numerical methods to integrate the two-body problem. The integration has been performed with Bettis methods, a symplectic algorithm and special spherically exact scheme. The results have been compared with those obtained with traditional integrators.
\end{abstract}

\section{INTRODUCTION}

The problem of choosing a suitable integrator from various numerical methods is quite a difficult task because their efficiency depends strongly on the dynamical nature of a particular dynamical system and the required accuracy.

In 1989 Kinoshita and Nakai ([7]) published the results of a questionnaire they sent to members of IAU Comission 7 (Celestial Mechanics) to check what kind of integrators they use. The answers show that classical methods such as 1 inear multistep algorithms: Adams-Bashforth, Adams-Moulton, Störmer, Cowel 1,... or Runge-Kutta methods are widespread. Other using the idea of Taylor series or similar ones are al so popular. Although much effort has been made in the last 20 years to develop more efficient special algorithms to be used in Celestial Mechanics problems, the aforementioned enquiry shows that many of the special codes are not much known and their use is not extended.

Sometimes it is not the efficiency but ot her practical conditions that determine which method is chosen. Classical 
methods have some advantages from the point of view of their usage. Almost all of them are implemented in popular soft ware packages easily available and ready to use. Besides, their merits and demerits have been much discussed and can be found in many textbooks. On the other hand, in most cases, special algorithms must be implement by the user. This is not so easy if we take into account that often the papers in which these methods appear do not give their explicit form. Perhaps that could be the reason why they have so little diffusion and are scarcely applied.

Nevertheless, we are of the opinion that special algorithms that can be used with less effort do exist. In this work we have chosen a few special methods and compared the results when applied to the two-body problem. This, we think, can contribute to shedding some light on the problem of criteria for selection of integrators.

Besides the special algorithms, we have used classical finite difference methods, more precisely a predictor-corrector scheme where the predietors are explicit Adams-Bashforth and the correctors implicit Adams-Moulton. Among the traditional integrators the Adams schemes are reputed to be highly efficient and much documentation exists on the subject. The selected special methods are symplectic integrators, Bettis algoriths and spherically exact methods.

Symplectic or canonical integrators are numerical integration schemes for Hamiltonian systems, which conserve the symplectic 2-form exactly; as a consequence, the numerical solutions have a property of area-preserving. Another important property is that they do not have a secular term in the discretization error of the energy integral. Recently some sympiectic integrators have been developed ([8],[12]), and also it has been proved ([12]) that some Runge-Kutta schemesGauss-Legendre, for instance - are canonical. Many works on the subject are appearing lately, some of them by Celestial Mechanics authors. The symplectic integrators tested in this paper have some additional properties such a's conservation of of the angular momentum vector of the $n$-body problem or time reversibility; but they restrict to a Hamiltonian with the form $H(p, q)=V(p)+U(q)$. Their explicit expression appears in $[14], f . i$.

Together with this kind, of algorithms, another class of special integrators appl ying to problems Hamiltonian or not can be found. In 1969 Bettis [1] developed some non-standard methods, obtained as a modification of classical difference schemes, to solve numerically perturbed problems and satisfying the requirement that they produce the exact solution after perturbing terms are switched -0 ff $i, e$, these special methods integrate certain products of an ordinary pol ynomial and a Fourier polynomial without truncation error. The Bett is methods are becoming almost classic for some users. They can 
be handled easily, since they do not differ much from the traditional multistep ones. In fact it suffices to modify conveniently the last two coefficients. In 1990 an improved version of Bettis methods has been introduced by Ferrándiz and Novo ([3]), fitted for long-term prediction.

Besides, some kinds of geometrically designed algorithms can be applied to problems of Celestial Mechanics. In 1987 Ferrandiz and Pérez ( 4$]$ ) introduced the first spherically exact scheme and then developed a whole family of methods with the same property ([5]), that implies that the numerically computed points of the solution curve lying on the sphere do remain on it. That fact can be useful for the integration of unitary vectors or, in other cases, means that the energy is preserved. As those presented earlier, these methods have simple expressions so their implementation is not complicated although they are no longer 1 inear. They follow the line of those designed by Lambert and McLeod ([9]). These algoriths are of special interest for the author because our present work is being developed in this field.

\section{DESCR IPTION OF THE EXPERIMENTS}

In this section we summarize the results of the integration performed with the algorithms mentioned in the foriner section. Some figures are included representing the position

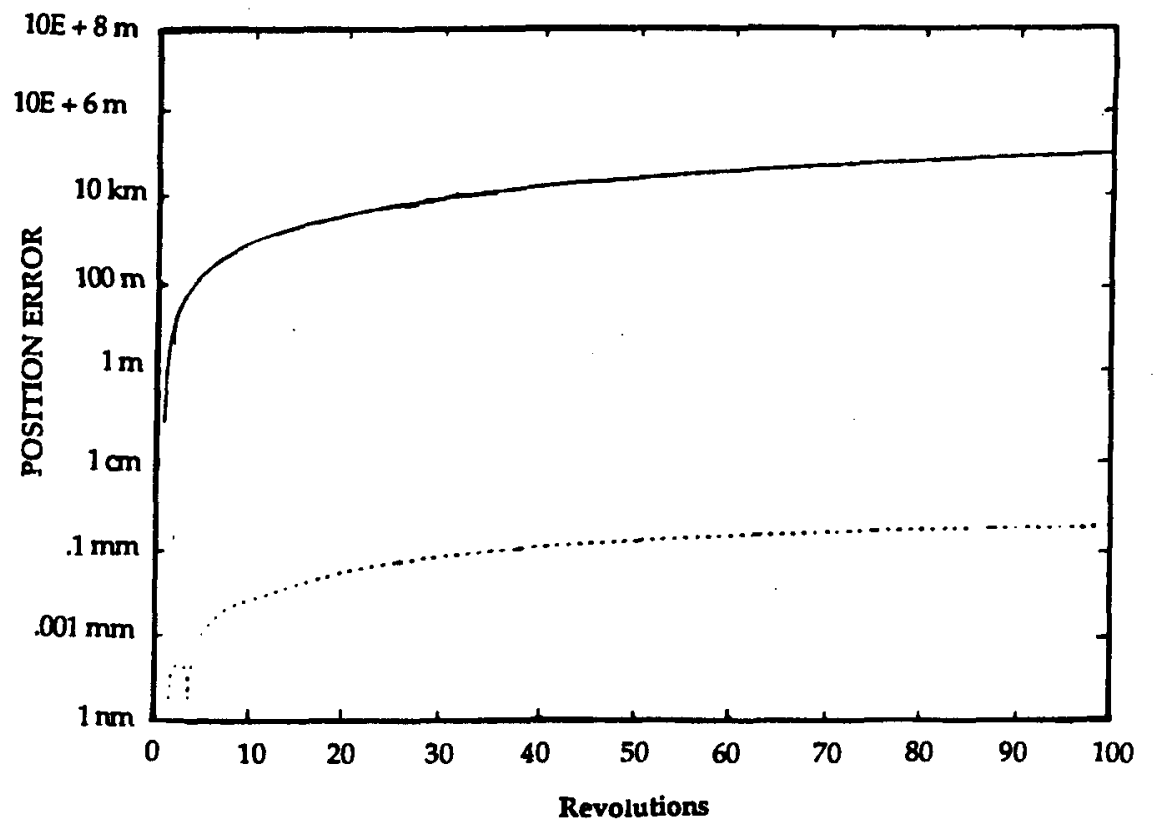

Figure 1:Kepler problem in Cartesian variables, e $=0, I=0$, order $=4$ 
error and the decimal logarith of the energy error versus the revolutions. The position errors measure the deviation of the solution computed with a desired number of steps per revolution from a reference orbit. This reference is a numericalisolution of the problem obtained with the same integrator. It has been selected satisfying strong requirements on conservation of $t h r e e$ independent integrals and stabilization of a number of digits in the computed solutions while the stepsize is being reduced. Nevertheless, its validity hast been assured using other classical integrators, more precisel y a Runge-Kutta-Fel hberg of adaptative stepsize.

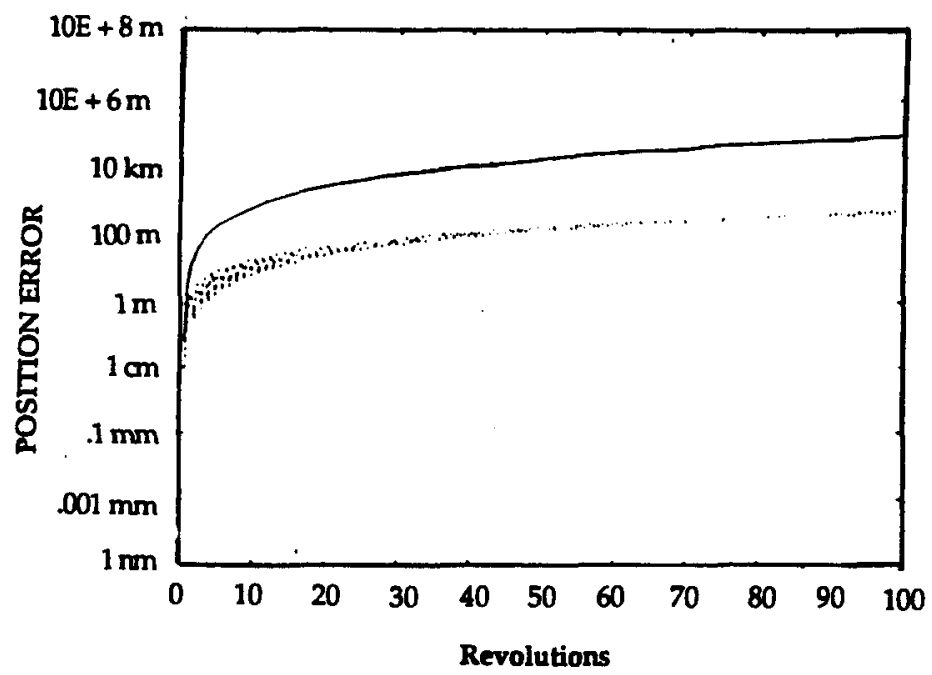

Figure 2: Main problem in Cartesian variables, $e=0, I=0$, $\mathrm{J} 2=0.001$, Order $=4$.

The special characteristics of the method's' fórce us to use different sets of variables. Thus, the required form of the Hamiltonian for the symplectic integrators oblige us to use Cartesian coordinates while for the spherically exact methods K-S variables are preferred. (see [5],[6]). Thus we have two different blocks of experiments.

In the first one, the two-body problem in Cartesian variables has been integrated by means of two symplectic integrators of orders four and six, two predictor-corrector pairs in PECE mode, -explicit Adams-Bashforth, implicit Adams-Moul ton- of order four and six and two Bettis schemes corresponding to the modification of the former Adams pairs. Notice that, although the equations of motion in Cartesian variables are not perturbed oscillators, the analitical expression of the solution for the circular Kepler problem allows to use successfully Bettis methods. The results with algorithms of the same order are compared in figures 1 to 4 . 


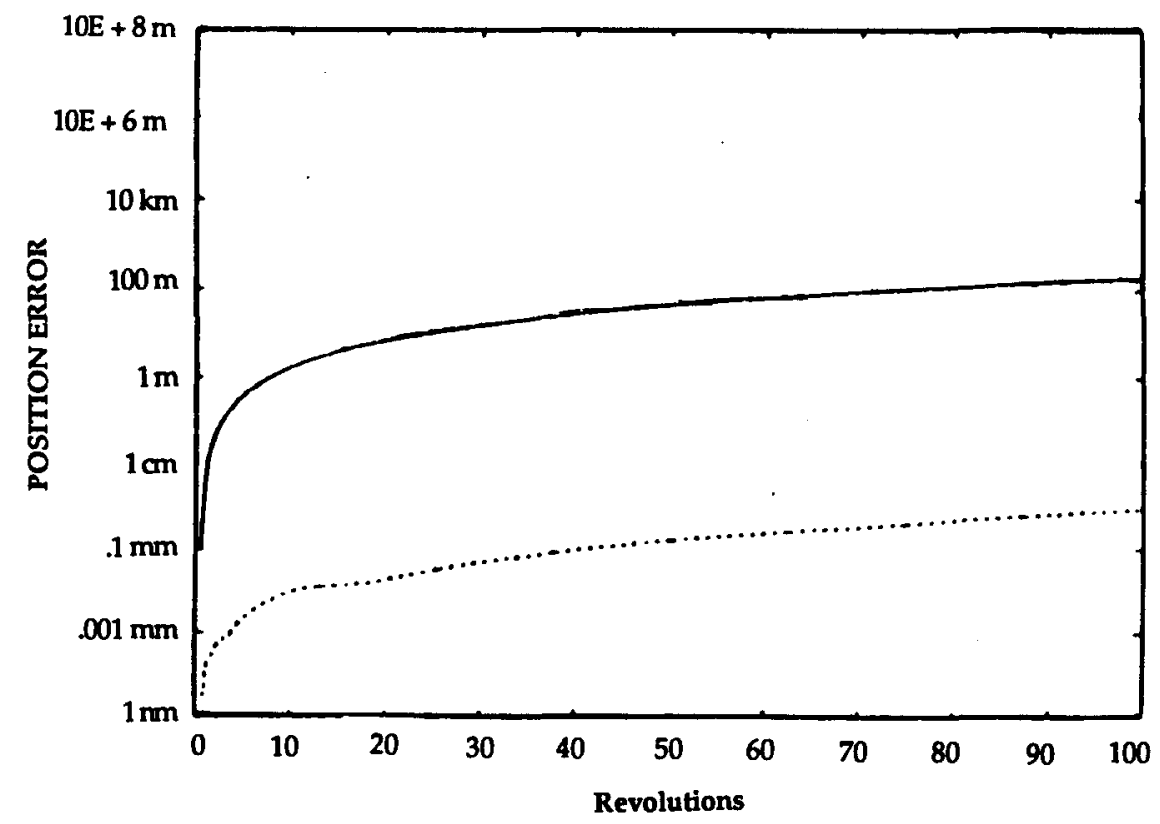

Figure 3: Kepler problem in Cartesian variables, $e=0, I=0$, Order $=6$.

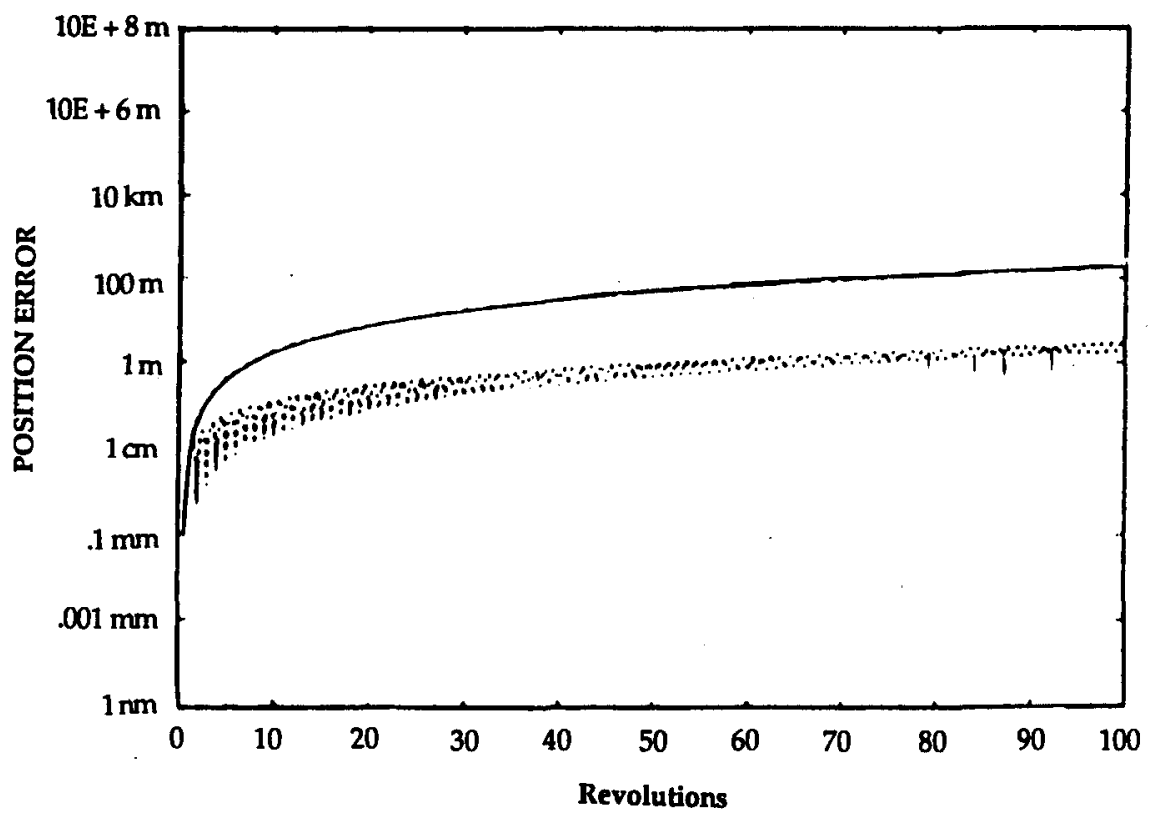

Figure 4: Main problem in Cartesian variables, $e=0, I=0$, $\mathrm{J} 2=0.001$, Order $=6$. 
The results of the integration of the Kepler problem with zero eccentricity and inclination are shown in figure 1 and figure 3 . In the first one the algorithms are of order four and in the second of order six. In this case the stepsize is fixed corresponding to 144 steps per revolution for Adams (solid) and Bettis (dashed) schemes. For the symplectic one (dotted) we have chosen the solution that needs the same number of evaluations of the function.

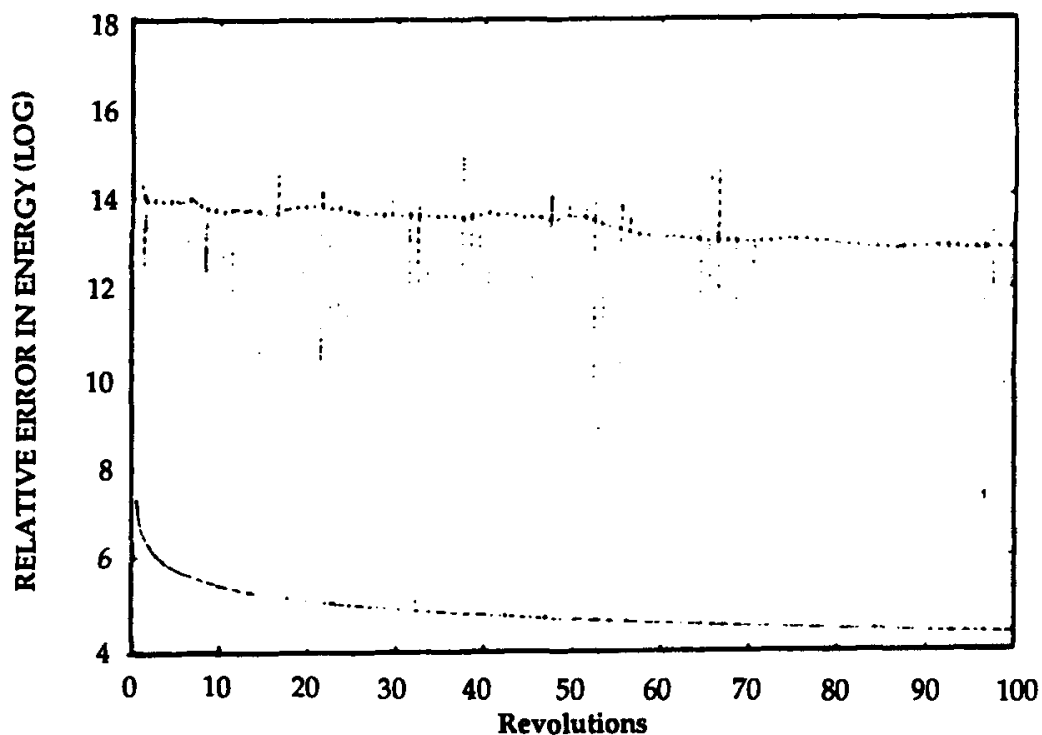

Figure 5: Kepler problem in Cartesian variables, $e=0, I=0$, Order $=4$.

In figures 2 and 4 the former problem has been perturbed with $J 2=0.001$. The rest of the conditions are the same as described in figure 1 and 3 , respectively.

Figures 5 to 8 show the opposite of the decimal logarithm of the relative error in energy versus the independent variable over a span of 100 revolutions performed in the four experiments described above. As before the solid graph corresponds to Adams, the dashed to Bettis and the dotted to the symplectic integrators.

The second block of experiments correspond to the Kepler problem in $K-S$ variables. As in the first group, it has been integrated with predictor-corrector pairs in PECE mode, both again of Adams type, but this time of order four and seven. The special algorithms used here are spherically exact ones (see [6]). They are non-l inear algor ithms obtained by means of a projection from the Adams. In this sense they are still predictor-corrector pairs in PECE mode. 


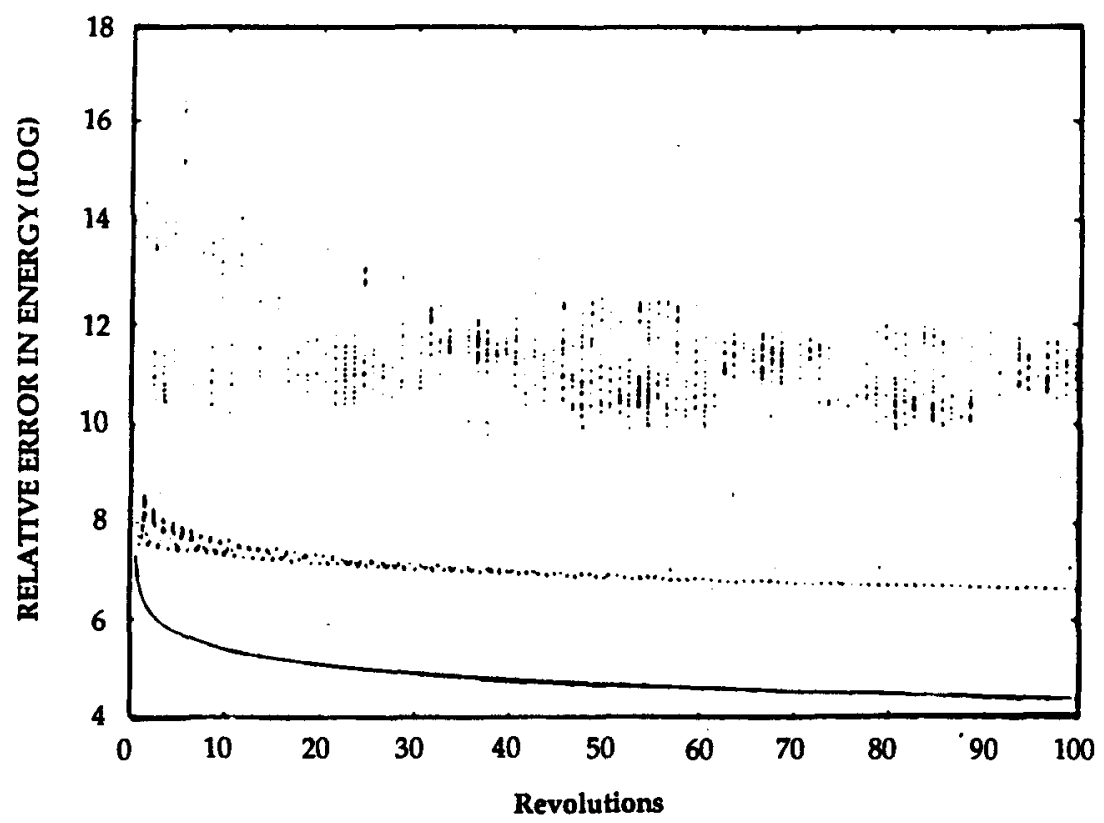

Figure 6: Main problem in Cartesian variables, $e=0, I=0$, $\mathrm{J} 2=0.001$, Order $=4$.

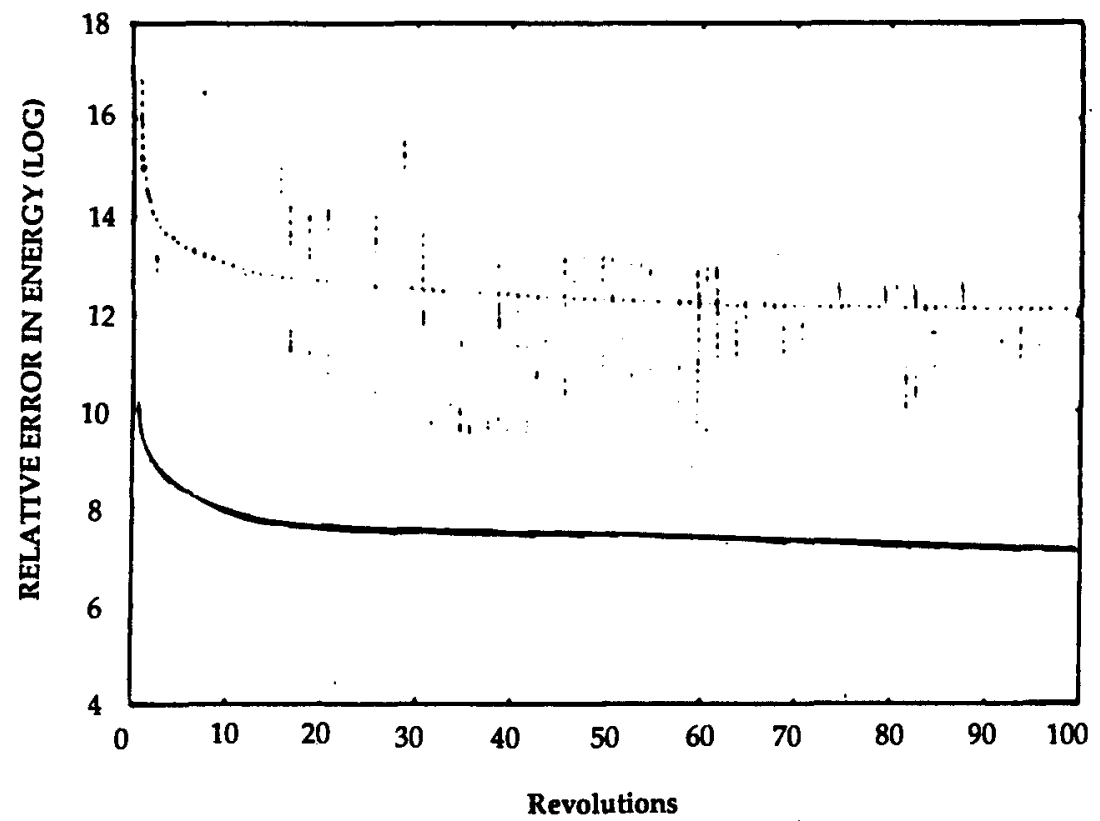

Figure 7: Kepler problem in Cartesian variables, $e=0, I=0$, Order $=6$. 


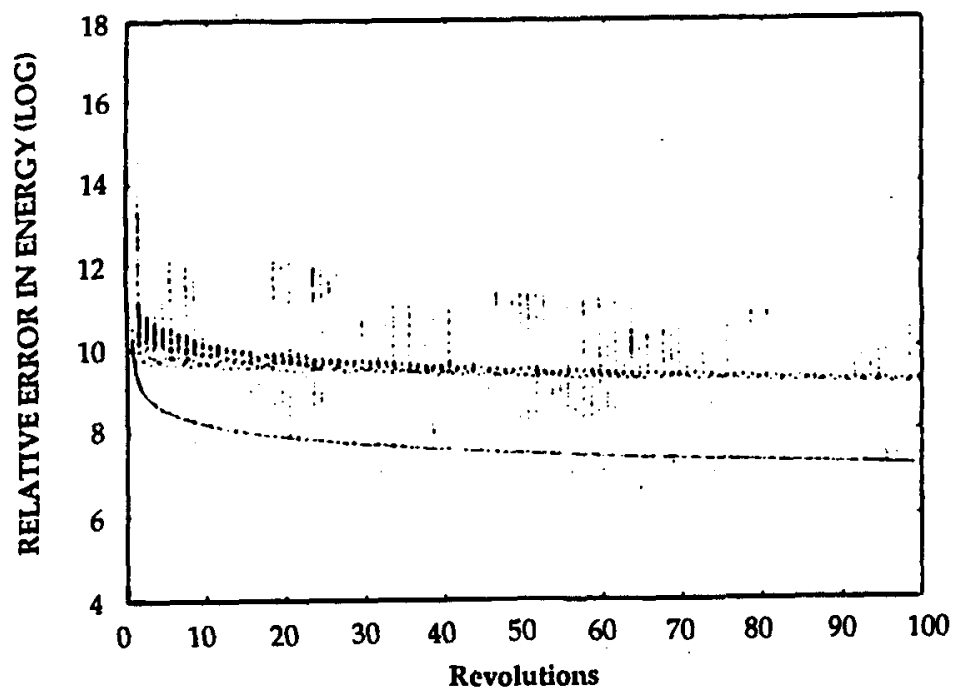

Figure 3: Main problem in Cartesian variables, $e=0, I=0$, $\mathrm{J} 2=0.001$, Order $=6$.

We do not want to give here an exhaustive study of the behaviour of these special integrators, but only to show some experiments that make feasible that further investigat ion could produce new more interesting fruits. More results concerning experimentation with spherically exact methods can be found in $[5],[6]$ or [11]

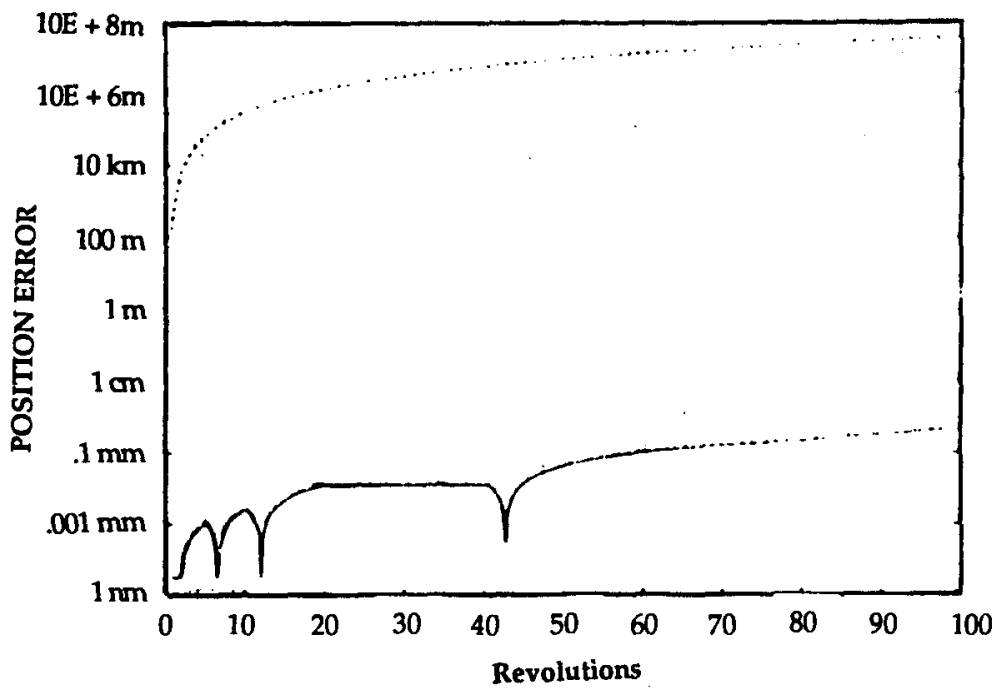

Figure 9: Kepler problem in $\mathrm{K}-\mathrm{S}$ variables, $e=0, \mathrm{I}=0,0$ order $=4$. 
In figures 9 and 10 the dashed and dotted upper curves represent the position error for the Adams integrator with 16 and 200 points per revolution, respectively. The lower solid graph shows the error of the spheric method for stepsize corresponding to only 16 points per revolution. The results for the last one are remarkable, since it performed almost a round $\rightarrow$ ff error while the classic Adams algorithm turned out to be highly inefficient for long stepsize and not very sat isfactory with smaller steps.

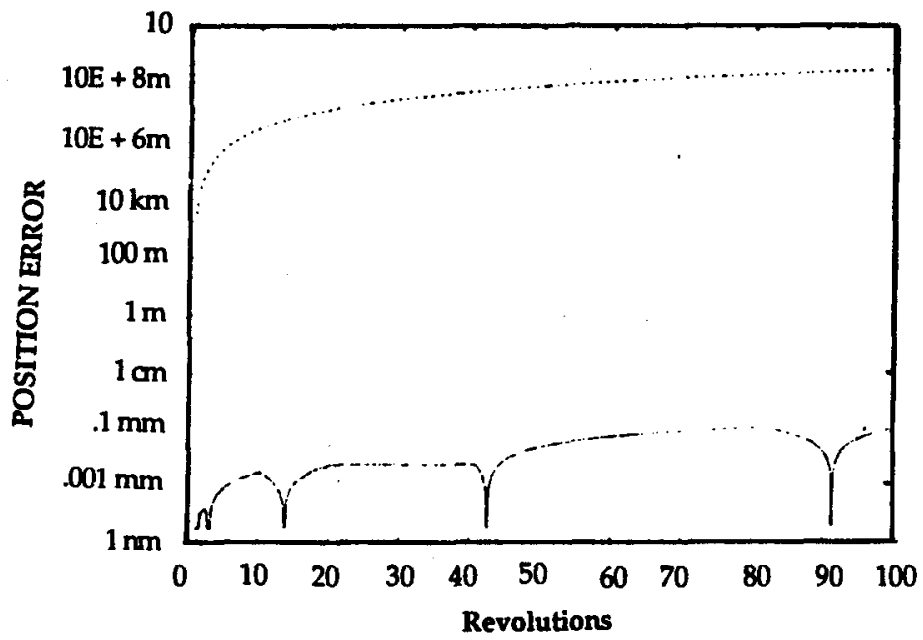

Figure 10: Kepler problem in $\mathrm{K}-\mathrm{S}$ variables, $e=0, \mathrm{I}=0$, Order $=7$.

The spherically exact methods preserves the energy as shown in figure 11 .

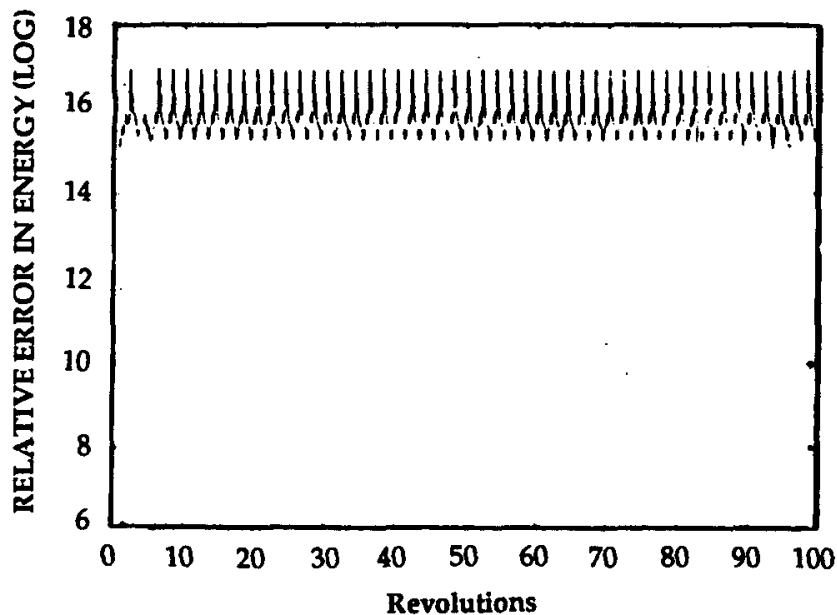

Figure 11: Kepl er problem in $K-S$ variables, $e=0, I=0$, Order $=4$. 


\section{FINAL REMARKS}

The Bettis algorithms have proved to be the most efficient of the three methods used to integrate the nearly circular two-body problem in Cartesian variables. Additional experimentation confirms this assertation and shows that they are more efficient than the Adams algorithms with a long stepsize, but this advantage is gradually lost when the stepsize is reduced. This behaviour is due to the $y$ they have been derived, which determines that when the stepsize tends to zero the modified coefficients of Bettis tend to those of Adams. These comments can be extended to all the cases where Bettis is applicable.

The symplectic integrators with the same cost give worse results than the linear schemes. The difference between both of them - notice that the error for the order four method, compared with Adams, is smaller than that for the six order one-can be explained by the distinct number of evaluation per step that they need. The first scheme evaluates $V(p)$ four $t$ imes and $U(q)$ three times as opposed to the ten and nine evaluations that the six order one requires, which means a high cost compared with the 1 inear schemes.

However, the error in energy exhibits a wide oscillation during each revolution, in such a way that, in general, the maximal errors are greater and the minimal ones are smaller than those given by the Bettis methods. In this case the conservation of the energy is not a good criterion for the error of position since a dephase can be observed in the integration. This agrees with the results of experiments shown in [8].

\section{ACKNO WL EDGEMENTS}

This work has been done as a part of the Project ESP. 88- 0541 from the CYCIT of Spain. The author acknowledges J.M. Ferrándiz for his continuous assistance and guidance and $S$.Novo for her help in numerical experinentation.

\section{REF ERENCES}

[1] Bettis, D.G.: 1970 , Numer. Math. 14,421-434.

[ 2 ] Ferrándiz, J.M.: 1988 , Clest. Mech. 41,343-357.

[3] Ferrándiz, J.M. and Novo, S.: 1990, in: Predictability, Stability and Chaos in N-body Dynamical Systems, A.E.Roy Ed., Plenum Publishing Corporation, NATO ASI Series C (in press).

[4] Ferrándiz, J.M. and Perez, M.T.: 1987, Actas del X C.E. D.Y.A., Valencia, Spain, pp. 105-9.

[5] Ferrándiz, J.M. and Perez, M.T., "A family of multi-step methods to integrate trajectories on spheres" (in

progress). 
[6] Ferrándiz, J.M. and Pérez, M.T.: 1990, in: Predictability, Stability and Chaos in $\mathrm{N}$-body Dynamical Systems, A.E.Roy Ed., Plenum Publishing Corporation, NATO ASI Series C (in press).

[7] Kinoshita, H., and Nakai, H.: 1989, Celest. Mech.45, 231-244.

[8] Kinoshita H., Yoshida, H. and Nakai, H.: 1990 Proceeding of the XXIII Symposium on "Celestial Mechanics", Kyoto, Japan, pp. $1-6$.

[9] Lambert, J.D. and UcLeod, R.J.Y.: 1979, Numerical Analysis Proceedings (Dundee), Springer Verlag 1980.

[10] Perez, M.T.: 1989, Doctoral Dissertation, Univ. Valladolid, Spain.

[11] Sanz-Serna, J.M.: 1988, BIT 28, 877-83.

[12] Stiefel, E., and Scheifele, G.: 1930, Linear and Regular Celestial Mechanics, Springer iVerrag.

[13] Yoshida, H.: 1990, Phys. Lett. (accepted). 